\title{
Crossing of Cyclotron and Spin Resonances in Two-Dimensional Coulomb Gas
}

\author{
Vladimir I. Fal'ko (a) \\ Max-Planck-Institut fur Festkorperforschung, Heisenbergstrasse 1, 7000 Stuttgart 80, Germany
}

(Received 2 November 1992)

\begin{abstract}
We predict the Shubnikov-de Haas oscillations of the fine structure of the cyclotron resonance near its crossing with the spin resonance in the degenerate two-dimensional Coulomb gas subjected to a strong tilted magnetic field. The spin-orbit coupling splits these resonances into three lines with the splitting gaps varying with the filling factor from $v_{\text {'so }} p_{F}$ to $v_{\text {'so }} p_{F} / \sqrt{2}$ at odd-and even-integer filling, respectively. This indicates reentrance of the system into a partially spin-polarized state and can be treated as manifestation of a new spin-density wave mode in it.
\end{abstract}

PACS numbers: $73.20 . \mathrm{Mf}$

Previous studies [1-6] of both the cyclotron resonance (CR) and electron spin resonance (ESR) in the twodimensional (2D) electron gas have confirmed the validity of Kohn's and Larmor's theorems for quantum systems with pronounced Coulomb correlations. That is, the analysis of the charge- and spin-density wave excitations has proved that the Coulomb and exchange effects in an ideally pure 2D system with a parabolic dispersion of electrons do not renormalize either the cyclotron or ESR frequencies, as compared to the single particle ones. More recent CR studies have shown that the above statement is conditioned by the features of a single particle spectrum itself. For example, in systems with a nonparabolic conduction band the detailed structure of the CR line is sensitive to the Coulomb correlations among carriers $[7,8]$ and does not coincide with those obtained from the scheme of nonequidistant Landau levels.

In the present paper we study the interplay between the electron-electron interaction in 2D electron gas (in a single ideally pure heterostructure or quantum well) and spin-orbit coupling (due to the lack of inversion symmetry in the system) under specific conditions of artificial degeneracy of spin-split Landau levels $[9,10]$. This situation can be realized in the $2 \mathrm{D}$ electron gas by subjecting it to a strongly tilted magnetic field so that this strong tilting makes up for the relative smallness of the electron $g$ factor [1 1,12] and provides $\omega_{c}=e H_{z} / m c \approx \omega_{s}=\mu g H / \hbar$ (let us assume $g>0$ ). In this case the degenerate Landau levels with different spins are resonantly mixed and then split in the first order on spin-orbital interaction [13], and the analysis below deals with the influence of the Coulomb correlations on the fine structure of the CR near its crossing with the ESR.

To anticipate a little, we can say that the electronelectron interaction has two important consequences which make this crossing different in the Coulomb and noninteracting gas. The first stems from the fact that the crossings CR-ESR and CR-combined frequency resonance (CFR) are resolved by the exchange interaction [3], and mixing and splitting involve different hybridized collective modes, as compared to free electrons [13].

Another feature of the interacting electron system consists of its transition to a collective spin-polarized state near the spin-split Landau levels crossing at even-integer filling factor, as predicted by Giuliani and Quinn [9] and probably observed by Koch et al. [12] in $\operatorname{In}_{x} \mathrm{Ga}_{1-{ }_{x}} \mathrm{As}-$ InP heterostructures. The exchange interaction of electrons stimulates the spin alignment, so that the electron gas at the CR-ESR crossing point has to be already polarized. The occupation of two excess Landau levels with the same spins produces a new spin-density wave mode which cannot manifest itself in ordinary spin resonance absorption but affects the fine structure of CR at its crossing with ESR, so that we are able to propose the way for its indirect observation.

In studying this problem, we use the approach of inter-Landau-level excitons [3,5,14]. This approach is applicable to completely filled 2D Landau levels and, formally, requires that the planar cyclotron energy $\hbar \omega_{c}$ be larger than the Coulomb energy $e^{2} / \chi \lambda_{H}$. It is based on the fact that the electrons from the filled Landau levels and the positive background of donors form a homogeneous neutral system [15] with chargeless excitations composed, as illustrated in Fig. 1, of an electron at one of the empty Landau levels [say, $N=(n, \alpha)$ ] correlated with a hole (empty state) at one of the completely filled ones $\left[N^{\prime}=\left(n^{\prime}, \alpha^{\prime}\right), \alpha=\downarrow\right.$ or $\left.\uparrow\right]$. As long as such excitations are neutral, their total momenta $\mathbf{Q}$ are exact quantum numbers, in spite of the presence of a quantizing magnetic field [3,5, I4]. After accounting for the spin degree of freedom (conserved in the Coulomb interaction), we get the basic set $\Psi_{N N^{\prime}}^{\dagger}(\mathbf{Q})|0\rangle$ of low-lying excited states where $\Psi_{N N^{\prime}}^{\dagger}(\mathbf{Q})$ are the creation and $\Psi_{N N^{\prime}}(\mathbf{Q})$ the annihilation operators of two particle excitations [16],

$$
\Psi_{N N^{\prime}}^{\dagger}(\mathbf{Q})=\sum_{p} e^{i p Q_{y}} a_{N p}^{\dagger} a_{N^{\prime} p}-Q_{x}
$$

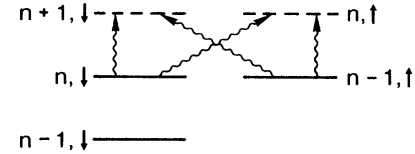

(a)

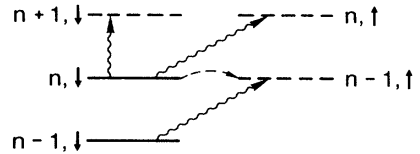

(b)
FIG. 1. Inter-Landau-level transitions involved in the exciton formation: (a) $v=2 n+1$; (b) $v=2 n$. 
In Eq. (1) we use the gauge $\mathbf{A}=\left(-H_{z} y+H_{y} z,-H_{x} z, 0\right)$ and the dimensionless momenta $Q$ and $p$ measured in units $1 / \lambda_{H}$ of the inverse magnetic length $\lambda_{H}=(\hbar c /$ $\left.e H_{z}\right)^{1 / 2} ;|0\rangle$ denotes the ground state.

In terms of the excitons $[3,5]$, we are allowed to replace the exact many-body Hamiltonian of 2D electrons, $H=H_{0}+V_{\text {so }}+U$, by the set of finite-size matrices,

$$
\begin{aligned}
H_{\left(\tilde{N} \tilde{N}^{\prime}\right)\left(N N^{\prime}\right)}= & \left\langle 0\left|\Psi_{\tilde{N} \tilde{N}^{\prime}}(\mathbf{Q}) H \Psi_{N N^{\prime}}^{\dagger}(\mathbf{Q})\right| 0\right\rangle \\
& -\delta_{N \tilde{N}} \delta_{N^{\prime} \tilde{N}^{\prime}}\langle 0|H| 0\rangle
\end{aligned}
$$

each acting in its own subspace of excitonic states with a fixed 2D momentum $\mathbf{Q}$ and total projection of a spin to the axis $\mathbf{h}=\mathbf{H} / H$. The pairs of Landau-level numbers $\left(N N^{\prime}\right)$ and $\left(\tilde{N} \tilde{N}^{\prime}\right)$ serve as indices of these matrices, and can be enumerated using the scheme of inter-Landaulevel transitions in Fig. 1. Each matrix has a blockdiagonal form with off-diagonal elements describing the conversion of one of the excitations, $\Psi_{N N^{\prime}}^{\dagger}(\mathbf{Q})|0\rangle$, into another, $\Psi_{\tilde{N} \tilde{N}^{\prime}}^{\dagger}(\mathbf{Q})|0\rangle$, in the course of Coulomb collisions or their mixing due to the spin-orbit coupling.

The single particle part $H_{0}=\sum \epsilon_{N} a_{N p}^{\dagger} a_{N p}$ of the Hamiltonian $H$ produces the diagonal terms in the matrix representation of Eq. (2). The spin-orbit interaction $V_{\text {so }}$ couples the charge- and spin-density wave excitations in the gas and under conditions of degeneracy of spin-split Landau levels has the form

$$
V_{\mathrm{so}}=\sum_{n, p} \sqrt{n+1}\left(a_{n \dagger p}^{\dagger} a_{n+1 \downarrow p} \alpha_{\mathrm{so}}+\text { H.c. }\right),
$$

where $\alpha_{\text {so }}=v_{\text {so }}(\mathbf{h}) \hbar / \lambda_{H}$ is defined in terms of the nominal spin-orbital velocity $v^{\prime}$ so $(h)$ (slightly modulated by the magnetic field orientation with respect to crystalline axes [13]).

In what follows, the spin-orbit coupling is treated as a weak perturbation which mixes a finite number of collective modes (with $\omega \approx \omega_{c}=\omega_{s}$ ) originally formed due to the electron-electron Coulomb interaction. The latter is accounted for in the Hamiltonian $H$ by the term

$$
\hat{U}=\frac{1}{2} \int d \mathbf{x} d \mathbf{x}^{\prime} \psi^{\dagger}(\mathbf{x}) \psi^{\dagger}\left(\mathbf{x}^{\prime}\right) \frac{e^{2}}{\chi\left|\mathbf{x}-\mathbf{x}^{\prime}\right|} \psi\left(\mathbf{x}^{\prime}\right) \psi(\mathbf{x})=\frac{1}{2} \int \frac{d \mathbf{q}}{(2 \pi)^{2}} \frac{2 \pi e^{2}}{\chi q \lambda_{H}}\left[\hat{\rho}^{\dagger}(\mathbf{q}) \hat{\rho}(\mathbf{q})-\hat{\rho}(0)\right],
$$

where $\chi$ is the effective dielectric constant of a media, and $\hat{\rho}(\mathbf{q})$ represents the Fourier transform of the electron density operator in terms of creation, $a_{N p}^{\dagger}$, and annihilation, $a_{N p}$, operators at Landau levels. To designate intermediate stages of calculations which give us the form of matrices in Eq. (2), we use definitions of Eq. (1), the algebra of operators $a_{N p}^{\dagger}$ and $a_{N^{\prime} p^{\prime}}$, and the fact that all Landau levels at zero temperature are either completely occupied or empty. This kind of calculation can be reformulated in the diagrammatic language, and we refer an interested reader to earlier works [3,14].

The case of the unit filling of the lowest Landau level, $v=1$, provides us with the most clear example, because there is a single magnetoplasma mode, $\omega_{\mathrm{pl}} \approx \omega_{c}+Q e^{2} /$ $2 \hbar \chi$, in the system which can be weakly coupled with a single spin exciton, $\omega(Q \rightarrow 0)=\omega_{s}[3,5]$. Therefore, the absorption peak near the crossing $\omega_{c}=\omega_{s}$ is split by the spin-orbit coupling into two peaks of equal intensities, $\omega_{ \pm}=\left(\omega_{c}+\omega_{s}\right) / 2 \pm\left[\left(\omega_{c}-\omega_{s}\right)^{2} / 4+\delta_{1}^{2}\right]^{1 / 2}$, which corresponds to the usual anticrossing structure of the CR-ESR degeneracy point. The splitting gap between peaks is $2 \delta_{1}$, $\delta_{1}=\left|\alpha_{\mathrm{so}} / \hbar\right|=p_{F} v_{\text {so }}(\mathbf{h})$, where $p_{F}$ is the zero-field Fermi momentum in the gas [17].

With higher odd-integer filling there are four degenerate excitons near the crossing, as shown in Fig. 1(a). Two of them correspond to transitions between neighboring Landau levels without change of a spin, which, nevertheless, does not indicate the coexistence of two independent cyclotron resonances in the system. The electromagnetic field can only excite them in the combination, $\Psi_{\mathrm{pl}}^{+}$, of the charge-density wave which has the linear dispersion $\omega_{\mathrm{pl}}(Q)=\omega_{c}+\pi \sigma_{x y} Q / \chi$ at small wave vectors coinciding with that of the classical magnetoplasma oscillations. Another mode, $\Psi_{\text {sd }}^{\dagger}$, is optically passive, but near CR-ESR crossing the spin-orbit coupling gets it mixed up with $\Psi_{n \nmid n \downarrow}^{\dagger}$ and $\Psi_{\mathrm{pl}}^{\dagger}$ excitations, since at $Q=0$ it is degenerate with them [3].

The reduced excitonic representation of the Hamiltonian $H(\mathbf{Q} \rightarrow 0)$ now takes the form of the $4 \times 4$ matrix,

$$
\left(\begin{array}{cccc}
\hbar \omega_{c} & 0 & \alpha_{\mathrm{so}}^{*} / \sqrt{2 n+1} & 0 \\
0 & \hbar \omega_{c} & -2 \alpha_{\mathrm{so}}^{*} \sqrt{n(n+1) /(2 n+1)} & \alpha_{\mathrm{so}} \sqrt{2 n+1} \\
\alpha_{\mathrm{so}} / \sqrt{2 n+1} & -2 \alpha_{\mathrm{so}} \sqrt{n(n+1) /(2 n+1)} & \hbar \omega_{s} & 0 \\
0 & \alpha_{\mathrm{so}}^{*} \sqrt{2 n+1} & 0 & \hbar\left(2 \omega_{c}-\omega_{s}\right)+E_{*}
\end{array}\right),
$$

which acts in the basis of states $\left\{\Psi_{\mathrm{pl}}^{\dagger}, \Psi_{\mathrm{sd}}^{\dagger}, \Psi_{n \dagger n \downarrow}^{\dagger}\right.$, $\left.\Psi_{n+1 \downarrow n-1 \uparrow}^{\dagger}\right\}|0\rangle$. Equation (5) shows that the electronelectron interaction shifts the energy of the combined frequency resonance mode $\Psi_{n+1 \downarrow n-1 \uparrow}^{\dagger}$ with respect to the single particle energy; hence the CR-ESR and CR-CFR crossings take place at different magnetic field tilting.
Since the additional exchange energy $E_{*}$ is negative (at $\left.v=3, E_{*}=-(\pi / 2)^{1 / 2} \frac{3}{16} e^{2} / \chi \lambda_{H}\right)$, the CR-CFR crossing occurs at $\omega_{c}-\omega_{s}=\left|E_{*} / \hbar\right|$, and if the spin-orbit coupling is weak enough the $4 \times 4$ matrix in Eq. (5) can then be replaced by two resonant $3 \times 3$ ones, each derived from $E q$. (5) near the corresponding crossing point [18]. 
The matrix Hamiltonian describing three inter-Landau-level excitons which are degenerate under the CRESR crossing conditions is given by the upper left $3 \times 3$ block in Eq. (5). In ideally pure systems the limit of $Q=0$ is an actual one, and we find the collective excitations spectrum as composed of the "unperturbed" cyclotron resonance mode, $\omega_{\mathrm{CR}}=\omega_{c}$, with the relative efficiency $I_{\mathrm{CR}} \propto 1-(2 n+1)^{-2}$, and two additional mixed modes with the frequencies

$$
\omega_{ \pm}=\frac{\omega_{c}+\omega_{s}}{2} \pm\left[\delta_{2 n+1}^{2}+\left(\frac{\omega_{c}-\omega_{s}}{2}\right)^{2}\right]^{1 / 2}
$$

and relative efficiencies $I_{ \pm}=0.5 /(2 n+1)^{2}$ at the crossing lat the lowest $v$ this is about $(5-10) \%$ of the total resonance intensity]. This gives us the unusual form of the fine structure of the CR near the crossing point shown in Fig. 2: Two chipped lines (satellites) which demonstrate the conventional anticrossing behavior coexist with the unperturbed $\mathrm{CR}$ line. At any odd-integer filling factor the splitting gaps between satellites,

$$
\delta_{2 n+1}=\sqrt{2 n+1}\left|\frac{\alpha_{\text {so }}}{\hbar}\right|=\frac{\sqrt{v} v_{\text {so }}(\mathbf{h})}{\lambda_{H}}=p_{F} v_{\text {so }}(\mathbf{h}),
$$

are equal to the zero magnetic field spin splitting [19] (determined by the $v_{\text {so }}$ and $p_{F}$ ) and are of the same magnitude as at $v=1$.

The analysis of even-integer filling factors is more complex because of the complexity of the ground state of the system close to the artificial degeneracy point. As compared to the previous case, the pair of degenerate Landau levels at the even-integer filling is "half filled," which gives freedom to their repopulation on approaching the crossing. It has been shown by Giuliani and Quinn [9] that the electron-electron interaction gives an earlier rise to the polarization transition in $2 \mathrm{D}$ gas stimulated by the inter-Landau-level exchange (i.e., at $\omega_{c}>\omega_{s}$ ) than one could expect from the single particle consideration. At $\omega_{c}=\omega_{s}$ the gas in an ideally pure $2 \mathrm{D}$ system at zero temperature forms the polarized and homogeneous ground state. A weak spin-orbit coupling (at $v=2$ it should be $\left|\alpha_{\text {so }}\right| \ll \frac{3}{16} E_{c}$, where $E_{c}=(\pi / 2)^{1 / 2} e^{2} / \chi \lambda_{H}[20]$, which requires $\left.v_{\text {so }} \ll e^{2} / \hbar \chi\right)$ does not change this result and perturbs the above ground state and elementary quasiparticles in it only slightly. This is to say that in ad-

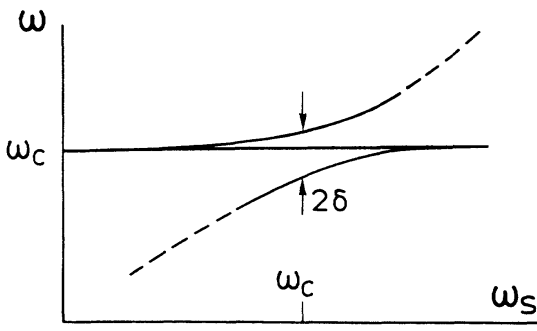

FIG. 2. The CR fine structure near the crossing. dition to a single cyclotron mode $\Psi_{2 \downarrow 1 \downarrow}^{\dagger}$, the polarized phase has two spin-flip excitation modes $\Psi_{0 \nmid 0 \downarrow}^{\dagger}$ and $\Psi_{1 \uparrow 1 \downarrow}^{\dagger}$ related to the spin flip at different Landau levels, as shown in Fig. 1(b). The block of the matrix Hamiltonian to describe these two is of the form

$$
\left(\begin{array}{cc}
\frac{1}{2} E_{c} & -\frac{1}{2} E_{c} \\
-\frac{1}{2} E_{c} & \frac{1}{2} E_{c}
\end{array}\right)
$$

and shows that real excitons are their symmetric and antisymmetric combinations, $\left(\Psi_{1 \dagger 1 \downarrow}^{\dagger} \pm \Psi_{0 \dagger 0 \downarrow}^{\dagger}\right) / \sqrt{2}$. The symmetric mode frequency is exactly equal (at $Q=0$ ) to the Zeeman splitting value, in agreement with Larmor's theorem. Only this mode can be excited by an external alternating magnetic field in the ESR experiments, whereas the new antisymmetric branch of spin +1 excitation with the energy $\epsilon(Q \rightarrow 0)=\hbar \omega_{s}+E_{c}$ cannot be excited in an ordinary spin resonance.

After this we can write the matrix Hamiltonian as

$$
\left(\begin{array}{cccc}
\hbar \omega_{c} & \alpha_{\text {so }} & (1+\gamma) \alpha_{\text {so }} & 0 \\
\alpha_{\mathrm{so}}^{*} & \hbar \omega_{s} & 0 & 0 \\
(1+\gamma) \alpha_{\mathrm{so}}^{*} & 0 & \hbar \omega_{s}+E_{c} & H_{12} \\
0 & 0 & H_{12}^{\dagger} & H_{22}
\end{array}\right),
$$

where $H_{12}$ describes the decay of the antisymmetric spin-density wave $\left(\Psi_{1 \uparrow 1 \downarrow}^{\dagger}-\Psi_{0 \dagger 0 \downarrow}^{\dagger}\right) / \sqrt{2}$ into a continuum of excitations composed of pairs of "cyclotron" $\left(\Psi_{2 \downarrow 1 \downarrow}^{\dagger}\right)$ and spin +1 low-energy $\left(\Psi_{0 \uparrow 1 \downarrow}^{\dagger}\right)$ excitons, and one can check [20] that the mode $\left.\Psi_{1 \uparrow 1 \uparrow}^{\dagger}+\Psi_{0 \dagger 0 \downarrow}^{\dagger}\right) / \sqrt{2}$ does not interact with the continuum spectrum, as it should follow from Larmor's theorem. Other off-diagonal terms in Eq. (8) owe their appearance to the spin-orbit coupling. The latter was taken into account in the first order both in the Hartree-Fock calculation of mixed single particle operators

$$
b_{(1,2) p}=x_{(1,2)} a_{1 \downarrow p}+y_{(1,2)} a_{0 \dagger p} \quad\left(b_{2 p}|0\rangle=0, b_{1 p}^{\dagger}|0\rangle=0\right)
$$

used in the excitons construction [this gives us corrections $\left.\gamma=\sqrt{2} / 4 E_{c} /\left(\frac{3}{4} E_{c}+\omega_{s}-\omega_{c}\right)\right]$ and in the coupling of pairs of higher Landau levels. After the Hartree-Fock procedure, the "spontaneous" creation of $\Psi_{b_{2} b_{1}}^{\dagger}$ excitons is forbidden in the lowest order on $\alpha_{\text {so }}$, and the cyclotron mode is also decoupled from two-excitonic states.

Although at $\omega_{c}=\omega_{s}$ the optically active cyclotron mode $\Psi_{2 \downarrow 1 \downarrow}^{\dagger}$ can be mixed only with a symmetric spin exciton, the interaction of these two is sufficiently affected just by the existence of the antisymmetric branch of spin-density waves. Indeed, this mode manifests itself in reduction by the factor of $1 / \sqrt{2}$ of ESR-CR coupling, since the direct spin-orbit interaction (initially, with the amplitude $\sqrt{2} \alpha_{\text {so }}$ ) exists only between $\Psi_{1 \uparrow 1 \downarrow}^{\dagger}$ and $\Psi_{2 \downarrow 1 !}^{\dagger}$ excitons and then has to be "equally shared" among symmetric and antisymmetric modes. The CR and SR near the crossing are, therefore, split into two lines with equal intensities and the gap $2 \delta_{2}$, where $\delta_{2}=\left|\alpha_{\text {so }}\right|$, is suppressed 
by the factor of $1 / \sqrt{2}$ as compared to those in Eqs. (6) and (7) for the odd-integer filling. This statement becomes obvious if one takes into account the relation between $\lambda_{H}$ and the zero-field Fermi momentum $p_{F}$ for a fixed filling and finds that $\delta_{2}=p_{F} v_{\text {so }}(\mathbf{h}) / \sqrt{2}$.

In extending our analysis to the polarized state at higher even-integer filling factors, we should account for one additional inter-Landau-level mode $\Psi_{n \uparrow n-1 \uparrow}^{\dagger}$ in the basis (see Fig. 1). This increases the number of excitons being in resonance, but the appropriate choice of magnetoplasma and spin-density waves reduces the problem to that considered. That is, in the vicinity of crossing the CR line acquires two weak satellites, similar to those at odd-integer filling, whereas the crossing of magnetoplasma modes $\Psi_{\mathrm{pl}}^{\dagger}$ and $\Psi_{\mathrm{sd}}^{\dagger}$ with antisymmetric spin +1 exciton can be ignored, because it occurs in the region of polarized phase instability. The CR acquires, therefore, the same fine structure as described by Fig. 2 with the only, though important, difference that the magnitude of the gaps $\delta_{2 n+2}$ between modes $\omega_{\mathrm{CR}}$ and $\omega_{ \pm}$,

$\delta_{2 n+2}=\sqrt{n+1}\left|\frac{\alpha_{\mathrm{so}}}{\hbar}\right|=\left(\frac{v}{2}\right)^{1 / 2} \frac{v_{\mathrm{so}}(h)}{\lambda_{H}}=\frac{p_{F} v_{\mathrm{so}}(\mathbf{h})}{\sqrt{2}}$,

is reduced by the factor of $1 / \sqrt{2}$ in comparison with those at odd-integer filling.

In summary, the proposed theory of the cyclotron resonance fine structure near its crossing with the electron spin resonance in the $2 \mathrm{D}$ Coulomb gas subjected to a strong tilted magnetic field shows that the CR is composed of three lines with the intensities evolving when the filling factor changes. One is just at the unperturbed cyclotron resonance frequency; its relative intensity increases from zero (at $v=1,2$ ) to almost $100 \%$ with occupation numbers higher than 3 . The other two are chipped by the spin-orbit interaction and their intensities undergo a reverse evolution. After combining the results obtained for the odd- and even-integer filling factors, we assume that the gaps which separate these split resonances are also filling factor dependent in such a way that they take the value of $v_{\text {so }} p_{F}$ or $v_{\text {so }} p_{F} / \sqrt{2}$ at $v=2 n+1$ and $v=2 n$, respectively, whereas the above parameter $v_{\text {so }} p_{F}$ (the zero-field spin splitting) is determined only by sheet electron density and the heterostructure material but is independent of the magnetic field. This kind of Shubnikov-de Haas oscillation in the CR spectrum manifests reentrance of the $2 \mathrm{D}$ system into a partially spinpolarized state and shows promise to indicate a new spin-density wave mode specific to this phase.

The author thanks K. Efetov, D. Heitmann, K. v. Klitzing, and A. MacDonald for discussions. This work was partially supported by the Alexander von Humboldt Foundation.

(a) On leave from Institute of Solid State Physics, Russian Academy of Sciences, Chernogolovka, 142432 Russia.

[1] W. Kohn, Phys. Rev. 123, 1242 (1961).

[2] N. Horing and M. Yildiz, Ann. Phys. 97, 216 (1976).

[3] C. Kallin and B. I. Halperin, Phys. Rev. B 30, 5655 (1984); 31, 3635 (1985).

[4] J. F. Janak, Phys. Rev. 178, 1416 (1969).

[5] Yu. A. Bychkov, S. V. Iordanskii, and G. M. Eliashberg, Pis'ma Zh. Eksp. Teor. Fiz. 33, 152 (1981) [JETP Lett. 33, 143 (1981)]; Yu. A. Bychkov and E. I. Rashba, Zh. Eksp. Teor. Fiz. 85, 1826 (1983) [Sov. Phys. JETP 58, 1062 (1983)].

[6] M. Dobers, K. v. Klitzing, and G. Weimann, Phys. Rev. B 38, 5453 (1988).

[7] Y. Takada and T. Ando, J. Phys. Soc. Jpn. 44, 905 (1978); J. Appel and A. W. Overhauser, Phys. Rev. B 18, 758 (1978).

[8] A. H. MacDonald and C. Kallin, Phys. Rev. B 40, 5795 (1989).

[9] G. F. Guiliani and J. J. Quinn, Phys. Rev. B 31, 6228 (1985); Surf. Sci. 170, 316 (1986).

[10] J.-W. Wu, P. Hawrylak, and J. J. Quinn, Phys. Rev. B 31, 6592 (1985).

[11] R. J. Nicholas et al., Phys. Rev. B 37, 1294 (1988).

[12] S. Koch et al., Phys. Rev. B 47, 4048 (1993).

[13] V. I. Fal'ko, Phys. Rev. B 46, 4320 (1992).

[14] I. V. Lerner and Yu. E. Losovik, Zh. Eksp. Teor. Fiz. 78, 1826 (1980) [Sov. Phys. JETP 51, 1062 (1980)].

[15] B. Janovici, Phys. Rev. Lett. 46, 386 (1981).

[16] Here and everywhere below we omit insufficient normalization factors from intermediate formulas.

[17] The magnitude of the splitting determines the requirement on the purity of our system: The CR linewidth (the Landau-level broadening) $\tau^{-1}$ should be less than the gap $\delta, \tau^{-1}<\delta$. We also assume a low enough temperature, $T<E_{c}, \hbar \omega_{c}$.

[18] The block related to CR-CFR crossing can be obtained from Eq. (5) by excluding terms corresponding to the exciton $\Psi_{n \nmid n \uparrow}^{\dagger}$. The CR mode is decoupled and is not affected by crossing with the CFR.

[19] G. Lommer, F. Malcher, and U. Rossler, Phys. Rev. Lett. 60, 728 (1988); B. Das, S. Datta, and R. Reifenberger, Phys. Rev. B 41, 8278 (1990); B. Jusserand et al., Phys. Rev. Lett. 69, 848 (1992).

[20] V. I. Fal'ko (unpublished). 